\title{
Estrogen-induced vitellogenin in Tor tambroides (Bleeker, 1854): purification, characterization and ELISA development.
}

\begin{abstract}
Vitellogenin (vtg) is a high molecular weight glycophospholipoprotein synthesized in the liver under stimulation of estrogen. Basically found in sexually mature female, vtg being taken up by developing oocyte during maturation. It functioned as a nutrient storage for growing embryo. Having potential to be used as a maturation indicator, vtg helps to enhance fish fry production. Tor tambroides is one of the most sought after fish in Malaysia for having potential as a game fish yet so important economically due to high demand by farmers as well as consumers. Main problem in the mass production of T. tambroides fry in hatchery is in the availability and selection of matured broodstock. Identification of matured and ready females morphologically can only be done by experience workers. Therefore, establishment of simple technique to definite identify matured females is necessary. This has led to the identification, purification and development of enzyme linked immunosorbent assay (ELISA) to measure blood plasma vtg as maturation indicator. This will definitely contribute to the hatchery production of $\mathrm{T}$. tambroides fry.
\end{abstract}

Keyword: Vitellogenin; Tor tambroides; ELISA; Estrogen. 